\title{
Fuzzy Reliability of Two Units of the Cold Storing System
}

\author{
Taotao Wang \\ Department of Science, Yanshan University \\ Qinhuangdao 066004, China \\ Xianyun Meng (Corresponding author) \\ Department of Science, Yanshan University \\ Qinhuangdao 066004, China E-mail:wangtaoqhd@163.com \\ Yanqin Guan \\ Department of Science, Yanshan University \\ Qinhuangdao 066004, China \\ Jianying Yang \\ Department of Science, Yanshan University \\ Qinhuangdao 066004, China
}

Supported by the Plan Projects of He Bei Education Office (No.2007323)

Supported by the Foundation for the natural science of He Bei province of China (A2005000301)

\begin{abstract}
This paper which adopts Probability statistics fuzzy mathematical principles and methods gives the Fuzzy reliability index of the cold storing system with two different units when the switch is completely reliable and the switch is not completely reliable (Switch life 0-1and Exponential distribution). And this paper gives a new kind of Failure mode, that is: system will be immediately failure if the switch is failure, meanwhile, it gives the new mode's Fuzzy reliability index.
\end{abstract}

Keywords: Cold storing system, Reliability, Fuzzy reliability

\section{Prior knowledge}

Knows $\mathrm{C}$ by literature [1] to express in the classical reliable definition "the product in...maintains its stipulation function" this clear event, $C_{1}, C_{2} \cdots C_{n}$ expressed separately each fuzzy function represent fuzzy event. Obviously $\mathrm{C}$ separately belongs to $C_{1}, C_{2} \cdots C_{n}^{\sim}$ in varying degrees. $C$ expresses the system breakdown, $C_{i}$ expressed "the ist unit is working", $C_{i}$ expressed the fuzzy function subset which we discussed.

By fuzzy conditional probability definition we obtain:

$$
\begin{aligned}
& P\left(C \Delta C_{j}\right)=P\left(C_{j} / C\right) \cdot P(C) \\
& P\left(C_{i} \Delta C_{j}\right)=p\left(C_{j} / C_{i}\right) \cdot P\left(C_{i}\right)
\end{aligned}
$$

According to the fuzzy reliable theory and the ordinary reliable theory we have:

$$
\begin{array}{lc}
P\left(C \Delta C_{j}\right)=R_{s} & P\left(C_{i} \Delta C_{j}\right)=R_{i} \\
P\left(C_{j} / C\right)=u c_{j}\left(R_{s}\right) & P\left(C_{j} / C_{i}\right)=u c_{j}\left(R_{i}\right)
\end{array}
$$

Substitutes (3) into (1) (2) we have:

$R_{s}=u c_{j}\left(R_{s}\right) \cdot R_{s}$ 


$$
R_{i}=u c_{j}\left(R_{i}\right) \cdot R_{i}
$$

Based on the literature [1], [4] knowledge, the relations between every unit fuzzy failure rate $\lambda_{i}$ and the ordinary failure rate $\lambda_{i}$ is:

$$
\underset{\sim}{\lambda_{i}}=\lambda_{i}-\frac{d u c_{j}\left(R_{i}\right) \cdot d t}{u c_{j}\left(R_{i}\right) d t}=\lambda_{i}-\overline{u^{\prime} c_{j}\left(R_{i}\right)}
$$

Where $\overline{u^{\prime} c_{j}\left(R_{i}\right)}$ is the relative rate of $u^{\prime} c_{j}\left(R_{i}\right)$.

\section{Fuzzy Reliability analysis}

Theorem 1 Suppose the system is the cold storing system with two different units and the switch is completely reliable, Its life respectively is $x_{1}, x_{2}$, also obeys separately exponential distribution $\lambda_{1}, \lambda_{2}$, mutually independent, so the fuzzy reliability and fuzzy mean lifetime are:

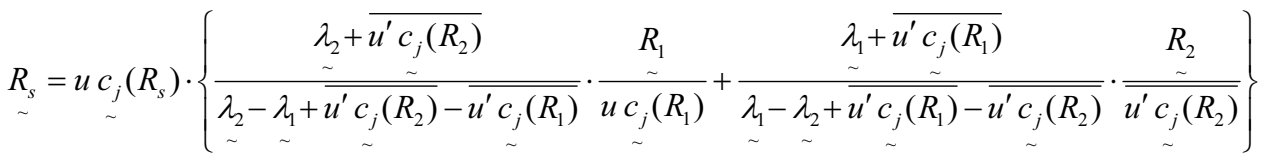

$$
\begin{aligned}
& \lambda_{1}+\lambda_{2}+\overline{u^{\prime} c_{j}\left(R_{1}\right)}+\overline{u^{\prime} c_{j}\left(R_{2}\right)}
\end{aligned}
$$

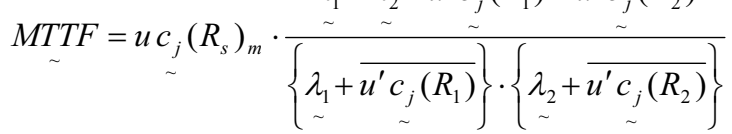

Proof: Known two unit life distributions respectively are $F_{1}=1-e^{-\lambda_{1} t}, F_{2}=1-e^{-\lambda_{2} t}$, also knows the system by literature [2] the reliability is:

$$
R_{s}=\frac{\lambda_{2}}{\lambda_{2}-\lambda_{1}} \cdot R_{1}+\frac{\lambda_{1}}{\lambda_{1}-\lambda_{2}} \cdot R_{2}
$$

So substitutes (5) (6) (7) into (4) we obtain the fuzzy reliability

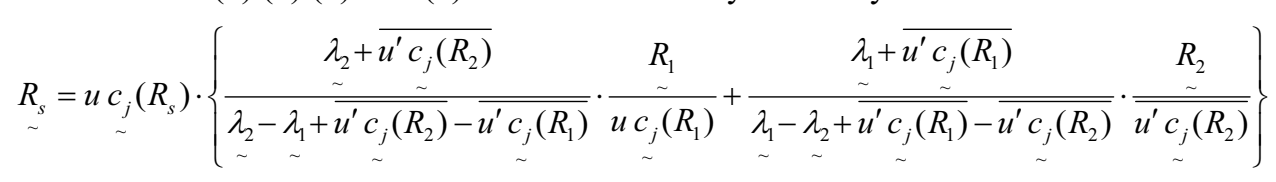

Result of $R_{s}=\frac{\lambda_{2}}{\lambda_{2}-\lambda_{1}} \cdot R_{1}+\frac{\lambda_{1}}{\lambda_{1}-\lambda_{2}} \cdot R_{2}$, obtain easily:

$$
\underset{\sim}{M T T F}=\int_{0}^{\infty} R_{s} d t=\int_{0}^{\infty} u c_{\sim}\left(R_{s}\right) \cdot R_{s} \cdot d t=u c_{\sim}^{c_{j}}\left(R_{s}\right)_{m} \cdot \int_{0}^{\infty} R_{s} \cdot d t
$$

$={ }_{u c_{j}}\left(R_{s}\right)_{m} \cdot \int_{0}^{\infty}\left[\frac{\lambda_{2}}{\lambda_{2}-\lambda_{1}} \cdot e^{-\lambda_{1} t}+\frac{\lambda_{1}}{\lambda_{1}-\lambda_{2}} \cdot e^{-\lambda_{2} t}\right] \cdot d t$

$=u c_{j}\left(R_{s}\right)_{m} \cdot\left[\frac{1}{\lambda_{1}}+\frac{1}{\lambda_{2}}\right]$

Therefore substitute (6) into equation (8), we have:

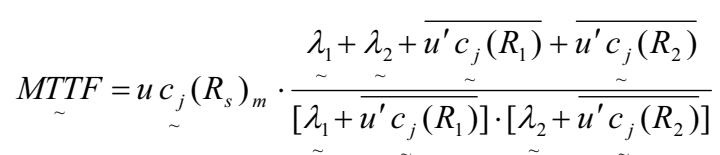

Where, $u c_{j}\left(R_{s}\right)_{m}-u c_{j}\left(R_{s}\right)$ is an average value which is in operating time sector

$[0, \infty)$, and it is a constant. 
Theorem 2 suppose the system is the cold storing system with two different units and the switch is not completely reliable, Its life respectively is $x_{1}, x_{2}$, Also obeys separately exponential distribution $\lambda_{1}, \lambda_{2}$, mutually independent, so the fuzzy reliability and fuzzy mean lifetime are:

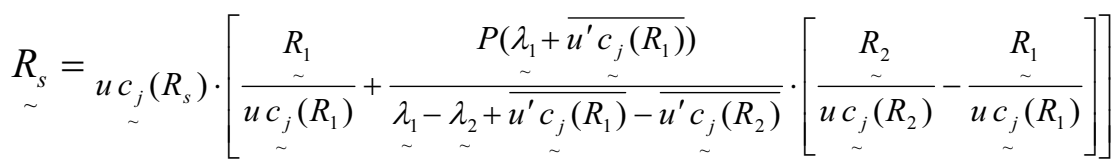

$$
\begin{aligned}
& \underset{\sim}{\operatorname{MTF}}=u c_{\sim}\left(R_{s}\right)_{m} \cdot\left[\frac{1}{\lambda_{\sim}+\overline{u^{\prime} c_{j}\left(R_{1}\right)}}+p \cdot \frac{1}{{\underset{\sim}{\sim}}_{\sim}+\overline{u^{\prime} c_{j}\left(R_{2}\right)}}\right]
\end{aligned}
$$

Proof: Introduces a random variable v, we have:

$$
p\{v=j\}=\left\{\begin{array}{l}
\mathrm{q}(\text { 当 } j=1) \\
p(\text { 当 } j=2)
\end{array}\right.
$$

The reliability of system is

$$
\begin{aligned}
R= & P\left\{\sum_{J=1}^{V} x_{j}>t\right\}=q \cdot p\left\{x_{1}>t\right\}+p \cdot p\left\{x_{1}+x_{2}>t\right\} \\
& =e^{-\lambda_{1} t}+\frac{p \lambda_{1}}{\lambda_{1}-\lambda_{2}}\left(e^{-\lambda_{2} t}-e^{-\lambda_{1} t}\right)=R_{1}+\frac{p \lambda_{1}}{\lambda_{1}-\lambda_{2}} \cdot\left(R_{2}-R_{1}\right)
\end{aligned}
$$

Substituting (10) (5) (6) into (4) entails that:

$$
\begin{aligned}
& R_{\sim}=u c_{\sim}\left(R_{s}\right) \cdot R_{s} \\
& =u c_{\sim}\left(R_{s}\right) \cdot\left[\frac{R_{1}}{\sim \underset{\sim}{u c_{j}\left(R_{1}\right)}}+\frac{P\left(\lambda_{1}+\overline{u^{\prime} c_{j}\left(R_{1}\right)}\right)}{\sim_{\sim}-\underset{\sim}{\sim} \lambda_{2}+\overline{u^{\prime} c_{j}\left(R_{1}\right)}-\overline{u^{\prime} c_{j}\left(R_{2}\right)}} \cdot\left[\begin{array}{c}
R_{\sim} \\
\frac{\sim}{u c_{j}\left(R_{2}\right)}-\frac{R_{1}}{u c_{j}\left(R_{1}\right)}
\end{array}\right]\right]
\end{aligned}
$$

Because the mean life of system is:

$$
M T T F=\int_{0}^{\infty} R_{s} d t=\int_{0}^{\infty} e^{-\lambda_{1} t}+\frac{p \lambda_{1}}{\lambda_{1}-\lambda_{2}}\left(e^{-\lambda_{2} t}-e^{-\lambda_{1} t}\right) d t=\frac{1}{\lambda_{1}}+p \frac{1}{\lambda_{2}}
$$

So the MTTF is:

$$
\begin{aligned}
\underset{\sim}{\operatorname{MTTF}}= & \int_{0}^{\infty} R_{s} d t=\int_{0}^{\infty} u c_{\sim}\left(R_{s}\right) \cdot R_{s} \cdot d t=u c_{j}\left(R_{s}\right)_{m} \cdot\left[\frac{1}{\lambda_{1}}+p \frac{1}{\lambda_{2}}\right] \\
& =\underset{\sim}{u c_{j}}\left(R_{s}\right)_{m} \cdot\left[\frac{1}{\lambda_{\sim}+\overline{u^{\prime} c_{j}\left(R_{1}\right)}}+p \cdot \frac{1}{\lambda_{\sim}+\overline{u^{\prime} c_{j}\left(R_{2}\right)}}\right]
\end{aligned}
$$

Theorem 3 The system not immediately expires when the switch is not working, the life of two different units is $x_{1}, x_{2}$, he life of switch is $x_{K}$, Obeys the exponential distribution separately and the parameter is $\lambda_{1}, \lambda_{2}$ and $\lambda_{K}$, mutually independent, so the fuzzy reliability and fuzzy mean lifetime are:

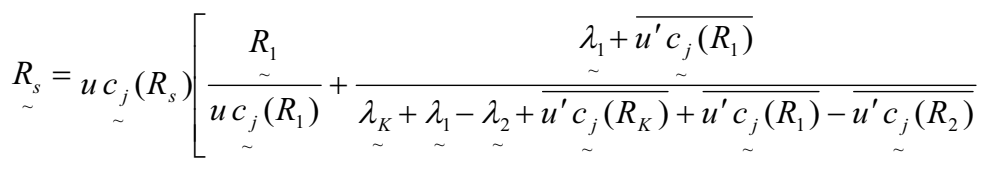

$$
\begin{aligned}
& \left.\cdot\left[\frac{R_{2}}{u c_{j}\left(R_{2}\right)}-\frac{R_{1}}{u c_{j}\left(R_{1}\right)} \cdot \frac{R_{K}}{u c_{j}\left(R_{K}\right)}\right]\right] \\
& M T T F=u c_{j}\left(R_{s}\right)_{m}
\end{aligned}
$$




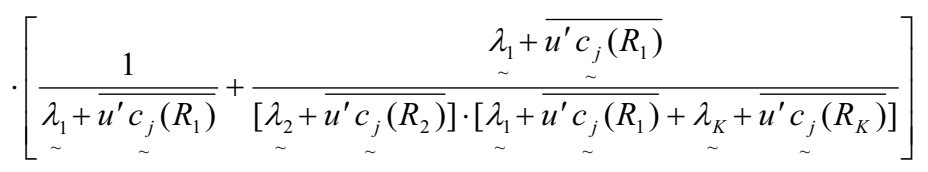

Proof: From literature [2] we know

$R=e^{-\lambda_{1} t}+\frac{\lambda_{1}}{\lambda_{K}+\lambda_{1}-\lambda_{2}}\left[e^{-\lambda_{2} t}-e^{-\left(\lambda_{1}+\lambda_{K}\right) t}\right]$

$$
\text { MTTF }=\frac{1}{\lambda_{1}}+\frac{\lambda_{1}}{\lambda_{2}\left(\lambda_{1}+\lambda_{K}\right)}
$$

Substituting (12) (5) (6) into (4) entails that

$$
R_{s}=u c_{j}\left(R_{s}\right) \cdot R_{s}
$$

$$
\begin{aligned}
& =u c_{j}\left(R_{s}\right)\left[\frac{R_{1}}{\frac{\sim}{u c_{j}\left(R_{1}\right)}}+\frac{\lambda_{1}+\overline{u^{\prime} c_{j}\left(R_{1}\right)}}{\underset{\sim}{\lambda_{K}+\lambda_{1}-\underset{\sim}{\lambda_{2}}+\overline{u^{\prime} c_{j}\left(R_{K}\right)}+\bar{\sim}} \overline{u^{\prime} c_{j}\left(R_{1}\right)}-\overline{u^{\prime} c_{j}\left(R_{2}\right)}}\right. \\
& \left.\cdot\left[\frac{R_{2}}{u c_{j}\left(R_{2}\right)}-\frac{R_{1}}{u c_{j}\left(R_{1}\right)} \cdot \frac{R_{K}}{u c_{j}\left(R_{K}\right)}\right]\right]
\end{aligned}
$$

Because also

$$
\begin{aligned}
\underset{\sim}{M T T F}= & \int_{0}^{\infty} R_{s} d t=\int_{0}^{\infty} u c_{\sim}\left(R_{s}\right) \cdot R_{s} \cdot d t \\
& =u c_{\sim}\left(R_{s}\right)_{m} \cdot \int_{0}^{\infty} R_{s} \cdot d t=u \underset{\sim}{c_{j}}\left(R_{s}\right)_{m} \cdot\left[\frac{1}{\lambda_{1}}+\frac{\lambda_{1}}{\lambda_{2}\left(\lambda_{1}+\lambda_{K}\right)}\right]
\end{aligned}
$$

Substituting (6) into (14) entails that

$$
M T T F=u c_{j}\left(R_{s}\right)_{m}
$$

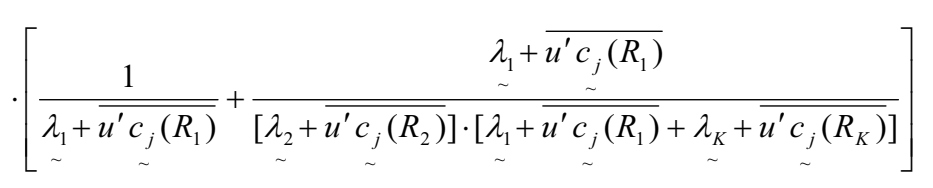

Theorem 4 The system immediately expires when the switch is not working, the life of two different units is $x_{1}, x_{2}$, the life of switch is $x_{K}$, Obeys the exponential distribution separately and the parameter is $\lambda_{1}, \lambda_{2}$ and $\lambda_{K}$, mutually independent, so the fuzzy reliability is:

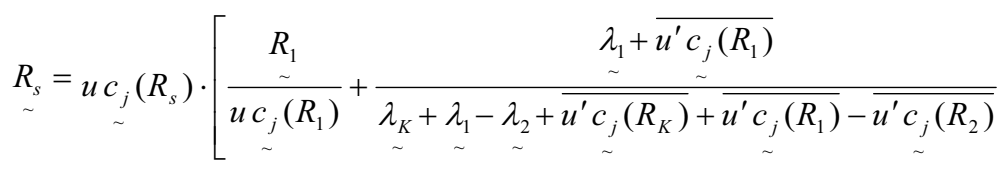

$$
\begin{aligned}
& \cdot\left[\frac{R_{2}}{u c_{j}\left(R_{2}\right)}-\frac{R_{1}}{u c_{j}\left(R_{1}\right)} \cdot \frac{R_{K}}{u c_{j}\left(R_{K}\right)}\right] \\
& {\left[\lambda_{K}+\overline{u^{\prime} c_{j}\left(R_{K}\right)}\right] \cdot\left[\lambda_{1}+\overline{u^{\prime} c_{j}\left(R_{1}\right)}\right]}
\end{aligned}
$$

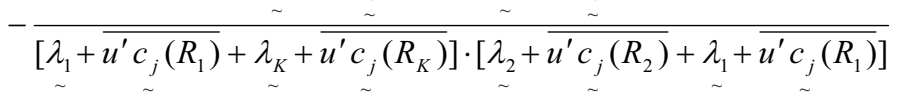

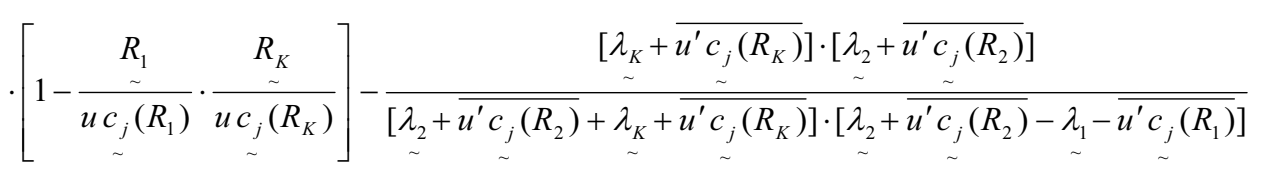




$$
\left.\cdot\left[1-\frac{R_{2}}{u c_{j}\left(R_{2}\right)} \cdot \frac{R_{K}}{u c_{j}\left(R_{K}\right)}\right]\right]
$$

Proof: the switch is not expire when the unit $x_{1}$ is not working, $x_{K}>x_{1}$, unit $x_{1}$ is replaced by storing unit $x_{2}$, the life of system is $x_{K}$ when unit $x_{2}$ is not expire.

Because the life distribution of system is:

$$
\begin{aligned}
1-R_{s}= & \iint_{t_{1} \leq t, t_{K} \leq t_{1}} \lambda_{1} \lambda_{K} e^{-\lambda_{1} t_{1}} e^{-\lambda_{K} t_{K}} d t_{1} d t_{K}+ \\
& \iiint_{t_{1}+t_{2} \leq t, t_{K}>t_{1}} \lambda_{1} \lambda_{2} \lambda_{K} e^{-\lambda_{1} t_{1}} e^{-\lambda_{2} t_{2}} e^{-\lambda_{K} t_{K}} d t_{1} d t_{2} d t_{K}+ \\
& \iiint_{t_{1}+t_{2} \geq t_{K}, t_{1} \leq t_{K} \leq t} \lambda_{1} \lambda_{2} \lambda_{K} e^{-\lambda_{1} t_{1}} e^{-\lambda_{2} t_{2}} e^{-\lambda_{K} t_{K}} d t_{1} d t_{2} d t_{K} \\
= & 1-e^{-\lambda_{1} t}-\frac{\lambda_{1}}{\lambda_{K}+\lambda_{1}-\lambda_{2}}\left[e^{-\lambda_{2} t}-e^{-\left(\lambda_{1}+\lambda_{K}\right) t}\right]+ \\
& \frac{\lambda_{K} \lambda_{1}}{\left(\lambda_{K}+\lambda_{1}\right)\left(\lambda_{2}-\lambda_{1}\right)}\left[1-e^{-\left(\lambda_{K}+\lambda_{1}\right) t}\right]+\frac{\lambda_{K} \lambda_{2}}{\left(\lambda_{K}+\lambda_{2}\right)\left(\lambda_{2}-\lambda_{1}\right)}\left[1-e^{-\left(\lambda_{K}+\lambda_{2}\right) t}\right]
\end{aligned}
$$

So the reliability of system is:

$$
\begin{aligned}
R_{s}= & e^{-\lambda_{1} t}+\frac{\lambda_{1}}{\lambda_{K}+\lambda_{1}-\lambda_{2}}\left[e^{-\lambda_{2} t}-e^{-\left(\lambda_{1}+\lambda_{K}\right) t}\right]- \\
& \frac{\lambda_{K} \lambda_{1}}{\left(\lambda_{K}+\lambda_{1}\right)\left(\lambda_{2}-\lambda_{1}\right)}\left[1-e^{-\left(\lambda_{K}+\lambda_{1}\right) t}\right]-\frac{\lambda_{K} \lambda_{2}}{\left(\lambda_{K}+\lambda_{2}\right)\left(\lambda_{2}-\lambda_{1}\right)}\left[1-e^{-\left(\lambda_{K}+\lambda_{2}\right) t}\right]
\end{aligned}
$$

Substituting (15) (5) (6) into (4) we obtain:

$$
\begin{aligned}
& R_{s}=u c_{j}\left(R_{s}\right) \cdot R_{s}
\end{aligned}
$$

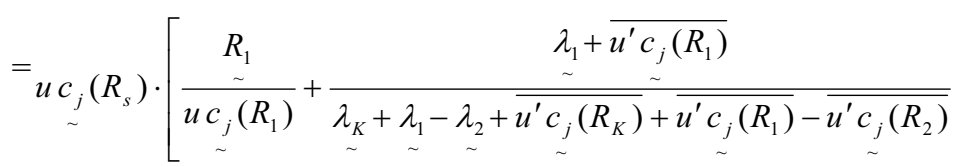

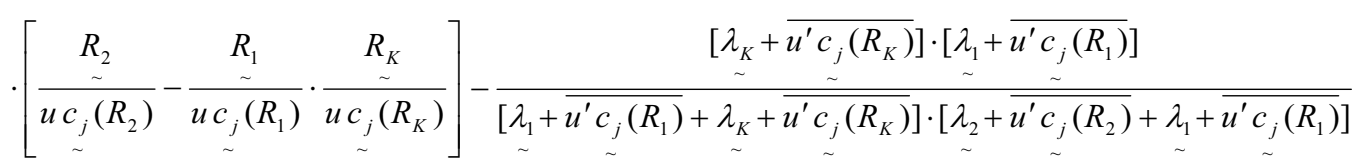

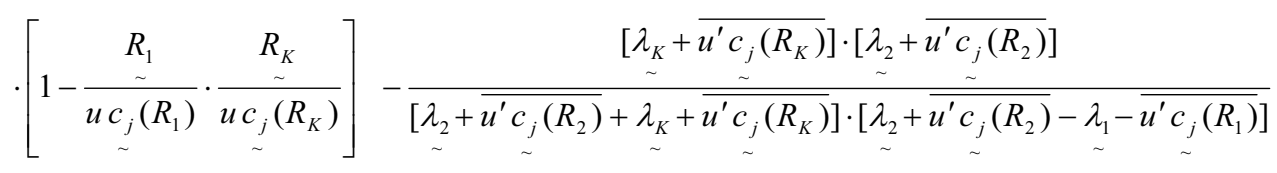

$$
\begin{aligned}
& \left.\cdot\left[1-\frac{R_{2}}{u c_{j}\left(R_{2}\right)} \cdot \frac{R_{K}}{u c_{j}\left(R_{K}\right)}\right]\right]
\end{aligned}
$$

\section{References}

Li, Tingjie\&Gao, He. Fuzzy Reliability. BUSEFAL. issue 35,1988.7.pp.12-25.

Cao, Jinhua \& Cheng, Kan. (2005). Introduction to Reliability Mathematics. Beijing: Science Press.pp. 45-52.

Huang, XiShi \&Leng, HuJi. Fuzzy Reliability of n Units of the Same Distribution Cool Storing System Mechanical Science and Technology for Aerospace Engineering, 1997.26(3). 25-27.

Miao, EnMing, Complementarity of cool reserve system reliability's mathematics model, Journal of Heilongjiang Institute of Science and Technology, 2003.13(4). 36-44. 
Meng, Xianyun, Yuan, Li \& Yin, Ruiling. The Reliability Analysis of a Two-unit Cold Standby System with failed Switch and Maintenance Equipment[A].Proceedings of the International Conference on Computational Intelligence and Security[C]. 2006. 941-944.

Meng, Xianyun, Li, Hongxia \&Li, Ning. Reliability Analysis of an n-unit Standby Repairable System with k Repair Facilities. Modern Applied Science.1(4). 55-59. 